УДК 591.87

\title{
Multiple Optima Identification Using Multi-strategy Multimodal Genetic Algorithm
}

\author{
Evgenii A. Sopov* \\ Informatics and Telecommunications Institute \\ Siberian State Aerospace University \\ Krasnoyarsky Rabochy, 31, Krasnoyarsk, 660037
}

Russia

Received 11.01.2016, received in revised form 25.02.2016, accepted 22.03.2016

\begin{abstract}
Multimodal optimization (MMO) is the problem of finding many or all global and local optima. In this study, a novel approach based on a metaheuristic for designing multi-strategy genetic algorithm is proposed. The approach controls the interactions of many search techniques (different genetic algorithms for MMO) and leads to the self-configuring solving of problems with a priori unknown structure. The results of numerical experiments for classical benchmark problems and benchmark problems from the IEEE CEC competition on MMO are presented. The proposed approach has demonstrated efficiency better than standard niching techniques and comparable to advanced algorithms. The main feature of the approach is that it does not require the participation of the human-expert, because it operates in an automated, self-configuring way.
\end{abstract}

Keywords: multimodal optimization, self-configuration, genetic algorithm, metaheuristic, niching. DOI: 10.17516/1997-1397-2016-9-2-246-257

\section{Introduction}

Many real-world problems have more than one optimal solution, or there exists only one global optimum and several local optima in the feasible solution space. Such problems are called multimodal. The goal of multimodal optimization (MMO) is to find all optima (global and local) or a representative subset of all optima.

Evolutionary and genetic algorithms (EAs and GAs) demonstrate good performance for many complex optimization problems. EAs and GAs are also efficient in the multimodal environment as they use a stochastic population-based search instead of the individual search in conventional algorithms. At the same time, traditional EAs and GAs have a tendency to converge to the best-found optimum losing population diversity.

In recent years MMO have become more popular, and many efficient nature-inspired MMO techniques were proposed. Almost all search algorithms are based on maintaining the population diversity, but differ in how the search space is explored and how optima basins are located and identified over a landscape. The majority of algorithms and the best results are obtained for realvalued MMO problems [1]. The main reason is the better understanding of landscape features in the continuous search space. Thus many well-founded heuristics can be developed.

Unfortunately many real-world MMO problems are usually considered as black-box optimization problems and are still a challenge for MMO techniques. Moreover, many real-world problems

*evgenysopov@gmail.com

(c) Siberian Federal University. All rights reserved 
contain variables of many different types, including integer, rank, binary and others. In this case, usually binary representation is used. Unfortunately, there is a lack of efficient approaches for problems with binary representation. Existing techniques are usually based on general ideas of niching and fitness sharing. Heuristics from efficient real-valued MMO techniques cannot be directly applied to binary MMO algorithms because of dissimilar landscape features in the binary search space.

In this study, a novel approach based on a metaheuristic for designing multi-strategy MMO GA is proposed. Its main idea is to create an ensemble of many MMO techniques and adaptively control their interactions. Such an approach would lead to the self-configuring solving of problems with a priori unknown structure.

\section{Related work}

The problem of MMO has exists since the first EAs. The first MMO techniques were applied in EAs and GAs for improvement in finding the global optimum in the multimodal environment.

The MMO, in general, can have at least 3 goals [2]:

- to find a single global optimum over the multimodal landscape only;

- to find all global optima;

- to find all optima (global and local) or a representative subset of all optima.

It is obvious that the second and the third goals are more interesting from both a theoretical and a practical point of view. At the same time, traditional search techniques have a tendency to converge to the best-found optimum losing population diversity, thus they are not efficient for solving MMO problems. An example of population convergence for standard and MMO algorithms are presented on Fig. 1.

Over the past decade interest for this field has increased. The recent approaches are focused on the goal of exploring the search space and finding many optima to the problem. Many efficient algorithms have been proposed. In 2013, the global completion on MMO was held within IEEE CEC'13 [3].

The list of widespread MMO techniques includes $[1,4,5]$ :

- General techniques: Niching (parallel or sequential), Fitness sharing, Clearing and Clusterbased niching, Crowding and Deterministic crowding, Restricted tournament selection (RTS), Mating restriction, Species conservation;

- Special techniques: Niching memetic algorithm, Multinational EA, Bi-objective MMO EA, Clustering-based MMO EA, Population-based niching, Topological algorithms;

- Other nature-inspired techniques: PSO, ES, DE, Ant Colony Optimization and others.

Binary and binarized MMO problems are usually solved using the GA based on general techniques. Also special techniques are applied, but some of their features can be lost in the binary space. Unfortunately, many efficient nature-inspired MMO algorithms have no binary version and cannot be easily converted to binary representation.

As we can see from many studies, there is no universal approach that is efficient for all MMO problems. Many researches design hybrid algorithms, which are generally based on a 
combination of search algorithms and some heuristic for niching improvement. For example, here are four top-ranked algorithms from the CEC'13 competition on MMO: Niching the CMAES via Nearest-Better Clustering (NEA2), A Dynamic Archive Niching Differential Evolution algorithm (dADE/nrand/1), CMA-ES with simple archive (CMA-ES) and Niching Variable Mesh Optimization algorithm (N-VMO) [3].

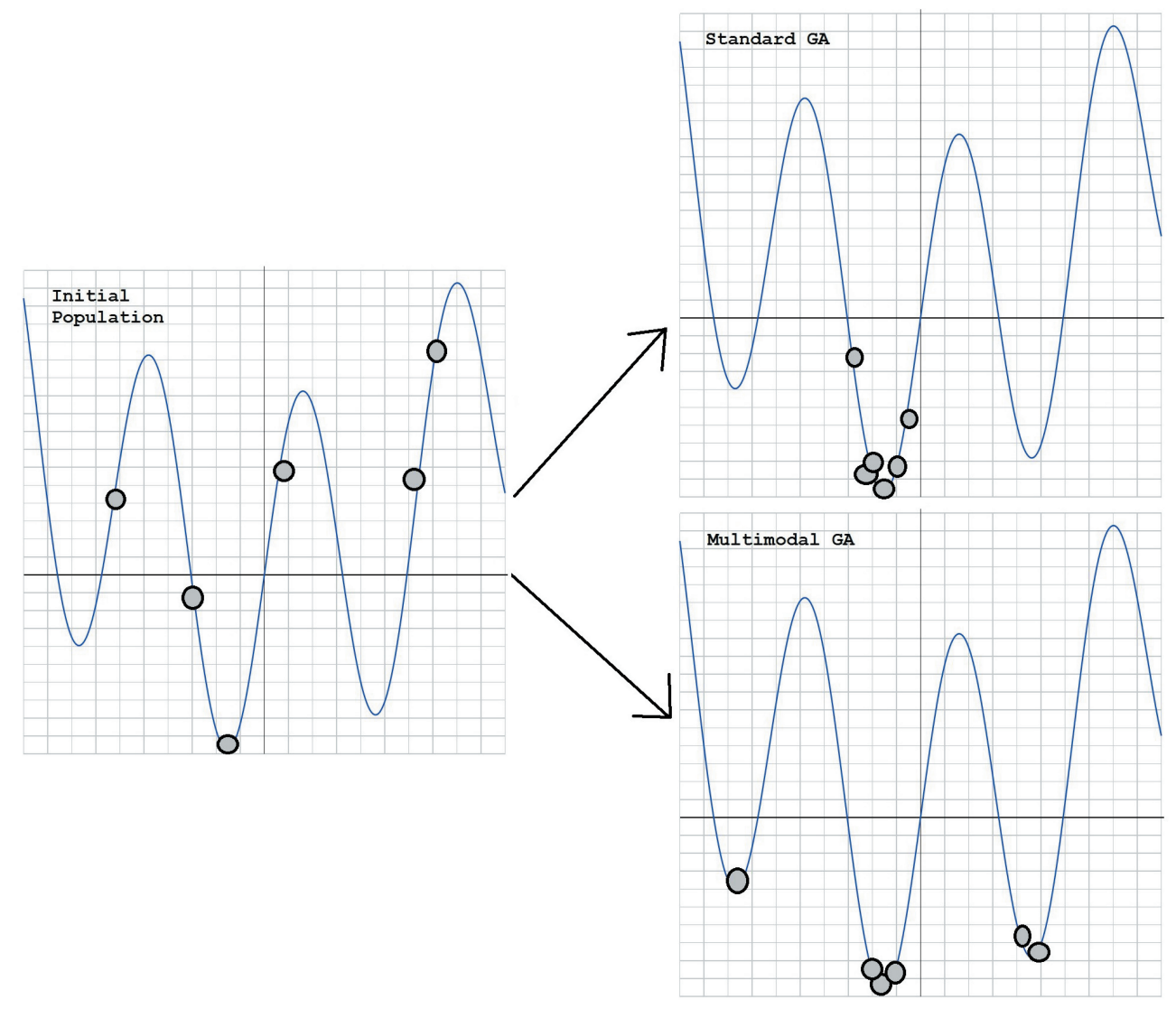

Fig. 1. Standard GA vs. Multimodal GA

Another way is combining many basic MMO algorithms to run in parallel, migrate individuals and combine the results. In [6] an island model is applied, where islands are iteratively revised according to the genetic likeness of individuals. In [7] four MMO niching algorithms run in parallel to produce offspring, which are collected in a pool to produce a replacement step. In [8] the same scheme is realized using the clearing procedure.

The conception of designing MMO algorithms in the form of an ensemble seems to be perspective. A metaheuristic that includes many different MMO approaches (different search strategies) can deal with many different MMO problems. And such a metaheuristic can be self-configuring due to the adaptive control of the interaction of single algorithms during the problem solving.

In [9] a self-configuring multi-strategy genetic algorithm in the form of a hybrid of the island model, competitive and cooperative coevolution was proposed. The approach is based on a parallel and independent run of many versions of the GA with many search strategies, which can deal with many different features of optimization problems inside the certain optimization class. 
The approach has demonstrated good results with respect to multi-objective and non-stationary optimization.

\section{Multi-strategy MMO GA}

In the field of statistics and machine learning, ensemble methods are used to improve decision making. On average, the collective solution of multiple algorithms provides better performance than could be obtained from any of the constituent algorithms. This concept can be also used in the field of EA. The main idea is to include different search strategies in the ensemble and to design effective control of algorithm interaction. Our hypothesis is that different EAs are able to deal with different features of the optimization problem, and the probability of all algorithms failing with the same challenge in the optimization process is low. Moreover, the interaction of algorithms can provide the ensemble with new options for optimization, which are absent in stand-alone algorithms.

The general structure of the self-configuring multi-strategy genetic algorithm proposed in [9] is called Self*GA (the star sign corresponds to the certain optimization problem) and it is presented in Fig. 2.

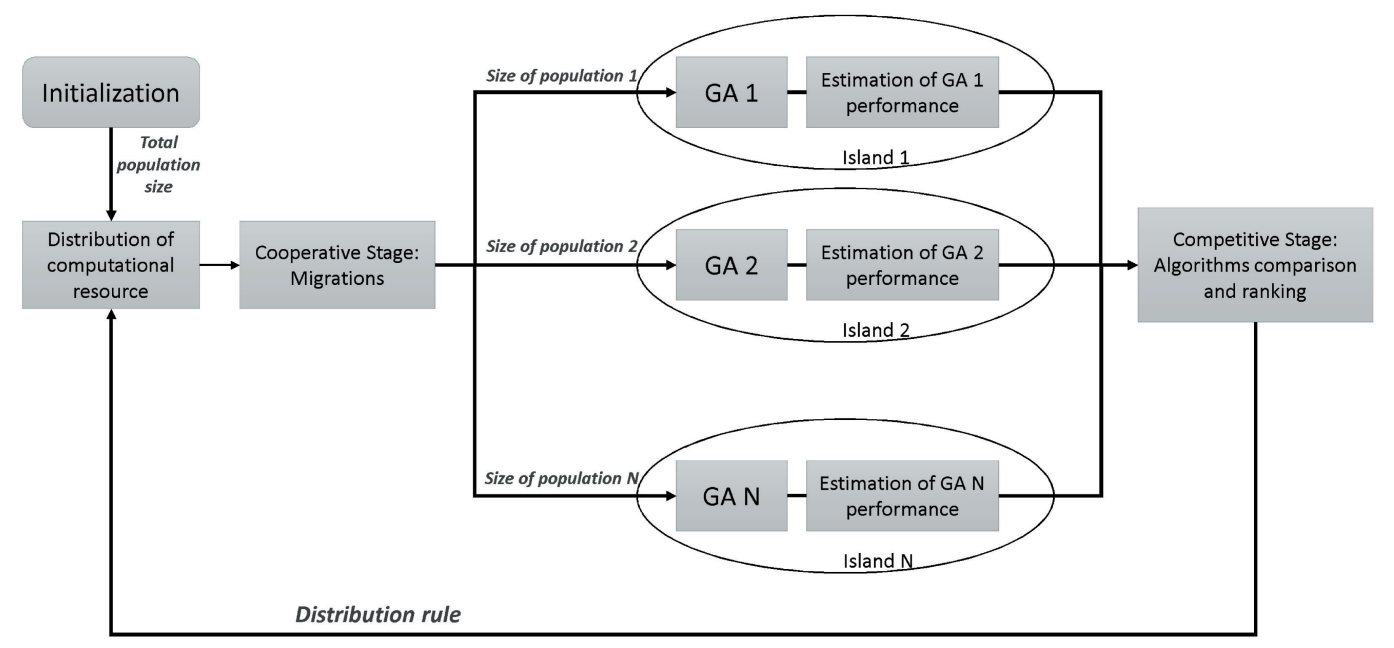

Fig. 2. The Self*GA structure

The total population size is called the computational resource. The resource is distributed between algorithms, which run in parallel and independent over the predefined number of iterations (called the adaptation period). After the distribution, each GA included in Self*GA has its own population which does not overlap with populations of other GAs. At the first iteration, all algorithms get an equal portion of the resource. This concept corresponds to the island model, where each island realizes its own search strategy.

After the adaptation period, the performance of individual algorithms is estimated with respect to the objective of the optimization problem. After that algorithms are compared and ranked. Search strategies with better performance increase their computational resource (the size of their populations). At the same time, all algorithms have a predefined amount of resource 
that is not distributed to give a chance for algorithms with low performance. This concept corresponds to the competitive coevolution scheme.

Finally, migrations of the best solutions are set to equate the start positions of algorithms for the run with the next adaptation period. According to the optimization problem, such a migration can be deterministic, selection-based or random. This concept corresponds to cooperative coevolution.

Such a technique eliminates the necessity to define an appropriate search strategy for the problem as the choice of the best algorithm is performed automatically and adaptively during the run.

Now we will discuss the design of a Self*GA for MMO problems that can be named SelfMMOGA. At the first step, we need to define the set of individual algorithms included in the SelfMMOGA. In this study we use six basic techniques, which are well-studied and discussed $[1,10]$, and they can be used with binary representation with no modification. Algorithms and their specific parameters are presented in Tab. 1. All values for radiuses and distances in Tab. 1 are in the Hamming metric for binary problems and in the Euclidean metric for continuous problems.

Table 1. Algorithms include in the SelfMMOGA

\begin{tabular}{|c|c|c|}
\hline Notation & Algorithm & Parameters \\
\hline \hline Alg1 & Clearing & Clearing radius, Capacity of a niche \\
\hline Alg2 & Sharing & Niche radius, $\alpha$ \\
\hline Alg3 & Clustering & $\begin{array}{c}\text { Number of clusters, min distance to centroid, } \\
\text { max distance to centroid }\end{array}$ \\
\hline Alg4 & $\begin{array}{c}\text { Restricted Tournament } \\
\text { Selection (RTS) }\end{array}$ & Window size \\
\hline Alg5 & Deterministic Crowding & - \\
\hline Alg6 & Probabilistic Crowding & - \\
\hline
\end{tabular}

The motivation of choosing certain algorithms is that if the SelfMMOGA performs well with basic techniques, we can develop the approach with more complex algorithms in further works.

The adaptation period is a parameter of the SelfMMOGA. Moreover, the value depends on the limitation of the computational resource (total number of fitness evaluations).

The key point of any coevolutionary scheme is the performance evaluation of a single algorithm. For MMO problems performance metrics should estimate how many optima were found and how the population is distributed over the search space. Unfortunately, good performance measures exist only for benchmark MMO problems, which contain knowledge of the optima. Performance measures for black-box MMO problems are still being discussed. Some good recommendations can be found in [11]. In this study, the following criteria are used.

The first measure is called Basin Ratio $(B R)$. The $B R$ calculates the number of covered basins, which have been discovered by the population. It does not require knowledge of optima, but an approximation of basins is used. The $B R$ can be calculated as 


$$
\begin{gathered}
B R(\text { pop })=\frac{l}{k}, \\
l=\sum_{i=1}^{k} \min \left\{1, \sum_{\substack{x \in p o p \\
x \notin z_{i}}} b\left(x, z_{i}\right)\right\}, \\
b\left(x, z_{i}\right)= \begin{cases}1, & x \in \operatorname{basin}(z), \\
0, & \text { otherwise, }\end{cases}
\end{gathered}
$$

where pop is the population, $k$ is the number of identified basins by the total population, $l$ is the indicator of basin coverage by a single algorithm, $b$ is a function that indicates if an individual is in basin $z$.

To use the metric (1), we need to define how to identify basins in the search space and how to construct the function $b(x, z)$.

For continuous MMO problems, basins can be identified using different clustering procedures like Jarvis-Patrick, the nearest-best and others [12]. In this study, for MMO problems with binary representation we use the following approach. We use the total population (the union of populations of all individual algorithms in the SelfMMOGA). For each solution, we consider a predefined number of its nearest neighbours (with respect to the Hamming distance). If the fitness of the solution is better, it is denoted as a local optima and the centre of the basin. The number of neighbours is a tunable parameter. For a real-world problem, it can be set from some practical point of view.

The function $b(x, z)$ can be easily evaluated by defining if individual $x$ is in a predefined radius of basin centre $z$. The radius is a tunable parameter. In this study, we define it as

$$
\text { radius }=\frac{\text { total population size }}{k},
$$

where $k$ is the number of identified basins $(k=|Z|)$.

The second measure is called Sum of Distances to Nearest Neighbour ( $S D N N)$. The $S D N N$ penalizes the clustering of solutions. This indicator does not require knowledge of optima and basins. The $S D N N$ can be calculated as

$$
\begin{aligned}
& S D N N(\text { pop })=\sum_{i=1}^{p o p \text { size }} d_{n n}\left(x_{i}, p o p\right), \\
& d_{n n}\left(x_{i}, p o p\right)=\min _{y \in p o p \backslash\{x\}}\{\operatorname{dist}(x, y)\},
\end{aligned}
$$

where $d_{n n}$ is the distance to the nearest neighbour, dist is the Hamming distance.

Finally, we combine the $B R$ and the $S D N N$ in an integrated criterion $K$ :

$$
K=\alpha \cdot B R(p o p)+(1-\alpha) \cdot \overline{S D N N}(p o p),
$$

where $\overline{S D N N}$ is a normalized value of $S D N N, \alpha$ defines weights of the $B R$ and the $S D N N$ in the sum $(\alpha \in[0,1])$.

Next, we need to design a scheme for the redistribution of computational resources. New population sizes are defined for each algorithm. In this study, all algorithms give to the "winner" algorithm a certain percentage of their population size, but each algorithm has a minimum 
guaranteed resource that is not distributed. The guaranteed resource can be defined by the population size or by problem features.

At the coopearative stage, in many coevolutionary schemes, all individual algorithms begin each new adaptation period with the same starting points (such a migration scheme is called "the best displaces the worst"). For MMO problems, the best solutions are defined by discovered basins in the search space. As we already have evaluated the approximation of basins $(Z)$, the solutions from $Z$ are introduced in all populations replacing the most similar individuals.

Stop criteria in the SelfMMOGA are similar to those in the standard GA: maximum number of objective evaluations, the number of generations with no improvement (stagnation), etc.

\section{Experimental results}

To estimate the approach performance we have used the following list of benchmark problems:

- Six binary MMO problems from [7]. These test functions are based on the unitation functions, and they are massively multimodal and deceptive.

- Eight real-valued MMO problems from IEEE CEC 2013 Special Session and Competition on Niching Methods for Multimodal Function Optimization [3].

We have denoted the functions as in the source papers. Some details of the problems are presented in Tab. 2. Real-valued problems have been binarized using the standard binary encoding with 5 accuracy levels.

Table 2. Test problem suite

\begin{tabular}{|c|c|c|c|c|c|}
\hline Problem & $\begin{array}{c}\text { Number of } \\
\text { desirable } \\
\text { optima }\end{array}$ & $\begin{array}{c}\text { Problem } \\
\text { dimensionality }\end{array}$ & Problem & $\begin{array}{c}\text { Number of } \\
\text { desirable } \\
\text { optima }\end{array}$ & $\begin{array}{c}\text { Problem } \\
\text { dimensionality }\end{array}$ \\
\hline \hline binaryF11 & 32 global & 30 & cecF2 & 5 global & $4,7,10,14,17$ \\
\hline binaryF12 & 32 global & 30 & cecF3 & $\begin{array}{c}1 \text { global } \\
\text { and 4 local }\end{array}$ & $4,7,10,14,17$ \\
\hline binaryF13 & 27 global & 24 & cecF4 & 4 global & $14,22,28,34,42$ \\
\hline binaryF14 & 32 global & 30 & cecF5 & $\begin{array}{c}2 \text { global } \\
\text { and 2 local }\end{array}$ & $11,17,24,31,37$ \\
\hline binaryF15 & 32 global & 30 & cecF6 & $\begin{array}{c}18 \text { global, } \\
742 \text { local }\end{array}$ & $16,22,30,36,42$ \\
\hline binaryF16 & 32 global & 30 & cecF7 & 36 global & $14,20,28,34,40$ \\
\hline cecF1 & $\begin{array}{c}2 \text { global } \\
\text { and 3 local }\end{array}$ & $9,12,15,19,22$ & cecF8 & 12 global & $8,14,20,28,34$ \\
\hline
\end{tabular}

The following criteria for estimating the performance of the SelfMMOGA over the benchmark problems are used for continuous problems:

- Peak Ratio $(P R)$ measures the percentage of all optima found by the algorithm (Equation 5). 
- Success Rate $(S R)$ measures the percentage of successful runs (a successful run is defined as a run where all optima were found) out of all runs.

$$
P R=\frac{\left|\left\{q \in Q \mid d_{n n}(q, p o p) \leqslant \varepsilon\right\}\right|}{k},
$$

where $Q=\left\{q_{1}, q_{2}, \ldots, q_{k}\right\}$ is a set of known optima, $\varepsilon$ is accuracy level.

The maximum number of function evaluation and the accuracy level for the $P R$ evaluation are the same as in CEC completion rules [3]. The number of independent runs of the algorithm is 50 .

In the case of binary problems, we cannot define the accuracy level in the $P R$, thus the exact points in the search space have to be found. This is a great challenge for search algorithms, thus we have substituted the $S R$ measure with Peak Distance $(P D)$. The $P D$ indicator calculates the average distance of known optima to the nearest individuals in the population [11].

$$
P D=\frac{1}{k} \cdot \sum_{i=1}^{k}\left(d_{n n}\left(q_{i}, p o p\right)\right) .
$$

The setting for the SelfMMOGA are:

- Maximum number of function evaluation is 50000 (as for the ENA);

- Total population size is 200 (the ENA uses 500);

- Adaptation period is 10 generations (25 times);

- All specific parameters of individual algorithms are self-tunable using the concept from [13].

We have estimated the $P R$, the $S R$ and the $P D$ averaged over 50 independent runs. We also have compared the results with Ensemble of niching algorithms (ENA) proposed in [7]. There is only the $S R$ value for the ENA. The experimental results show that binary problems are not too complex for the SelfMMOGA and the ENA. Differences in the performance estimation are not statistically significant, but the SelfMMOGA always outperforms the average of its stand-alone component algorithms for binary problems.

The results of estimating the performance of the SelfMMOGA with the pack of continuous problems are presented in Tabs. 3 and 4. We have compared the results of the SelfMMOGA runs with some efficient techniques from the competition. The techniques are $\mathrm{DE} / \mathrm{nrand} / 1 / \mathrm{bin}$ and Crowding DE/rand/1/bin [14], N-VMO [15], dADE/nrand/1 [16], and PNA-NSGAII [17]. Tab. 3 shows a comparison of average values with other techniques and Tab. 4 contains ranks of algorithms by separate criteria.

All problems and settings are as in the rules of the CEC'13 competition on MMO. For each problem there are 5 levels of accuracy of finding optima $\left(\varepsilon=\left\{10^{-1}, 10^{-2}, 10^{-3}, 10^{-4}, 10^{-5}\right\}\right)$. Thus, each problem have been binarized 5 times. The dimensionalities of binarized problems are presented in Tab. 2.

The settings for the SelfMMOGA are:

- Maximum number of function evaluation is 50000 (for cecF1-cecF5) and 200000 (for cecF6cecF8);

- Total population size is 200 ; 
- Adaptation period is 10 generations 25 times (for cecF1-cecF5) and 25 generations 40 times (cecF6-cecF8);

- All specific parameters of individual algorithms are self-tunable.

As we can see from Tabs. 3 and 4, the SelfMMOGA shows results comparable with popular and well-studied techniques. It yields to dADE/nrand/1 and N-VMO, but we should note that these algorithms are specially designed for continuous MMO problems, and have taken 2nd and 4th places, respectively, in the CEC competition. At the same time, the SelfMMOGA has very close average values to the best two algorithms, and outperforms PNA-NSGAII, CrowdingDE and DE, which have taken 7th, 8th and 9th places respectively.

Table 3. Average PR and SR for each algorithm

\begin{tabular}{|c|c|c|c|c|c|c|}
\hline \multirow{2}{*}{$\varepsilon$} & \multicolumn{2}{|c|}{ SelfMMOGA } & \multicolumn{2}{c|}{ DE/nrand/1/bin } & \multicolumn{2}{c|}{ cDE/rand/1/bin } \\
\cline { 2 - 7 } & PR & SR & PR & SR & PR & SR \\
\hline \hline $1 \mathrm{e}-01$ & 0,962 & 0,885 & 0,850 & 0,750 & 0,963 & 0,875 \\
\hline $1 \mathrm{e}-02$ & 0,953 & 0,845 & 0,848 & 0,750 & 0,929 & 0,810 \\
\hline $1 \mathrm{e}-03$ & 0,943 & 0,773 & 0,848 & 0,748 & 0,847 & 0,718 \\
\hline $1 \mathrm{e}-04$ & 0,907 & 0,737 & 0,846 & 0,750 & 0,729 & 0,623 \\
\hline 1e-05 & 0,816 & 0,662 & 0,792 & 0,750 & 0,642 & 0,505 \\
\hline Average & 0,916 & 0,780 & 0,837 & 0,750 & 0,822 & 0,706 \\
\hline \hline \multirow{2}{*}{$\varepsilon$} & \multicolumn{2}{|c|}{ N-VMO } & \multicolumn{2}{c|}{ dADE/nrand/1 } & \multicolumn{2}{c|}{ PNA-NSGAII } \\
\cline { 2 - 7 } & PR & SR & PR & SR & PR & SR \\
\hline \hline 1e-01 & 1,000 & 1,000 & 0,998 & 0,938 & 0,945 & 0,875 \\
\hline $1 \mathrm{e}-02$ & 1,000 & 1,000 & 0,993 & 0,828 & 0,910 & 0,750 \\
\hline $1 \mathrm{e}-03$ & 0,986 & 0,813 & 0,984 & 0,788 & 0,906 & 0,748 \\
\hline 1e-04 & 0,946 & 0,750 & 0,972 & 0,740 & 0,896 & 0,745 \\
\hline 1e-05 & 0,847 & 0,708 & 0,835 & 0,628 & 0,811 & 0,678 \\
\hline Average & 0,956 & 0,854 & 0,956 & 0,784 & 0,893 & 0,759 \\
\hline
\end{tabular}

Table 4. Algorithms ranking over cecF1-cecF8 problems

\begin{tabular}{|c|c|c|c|}
\hline $\begin{array}{c}\text { Rank by PR } \\
\text { criterion }\end{array}$ & Algorithm & $\begin{array}{c}\text { Rank by SR } \\
\text { criterion }\end{array}$ & Algorithm \\
\hline \hline 1 & $\begin{array}{c}\text { N-VMO and } \\
\text { dADE } / \text { nrand } / 1\end{array}$ & 1 & N-VMO \\
\hline 2 & SelfMMOGA & 2 & dADE $/$ nrand $/ 1$ \\
\hline 3 & PNA-NSGAII & 3 & SelfMMOGA \\
\hline 4 & DE $/$ nrand $/ 1 /$ bin & 4 & PNA-NSGAII \\
\hline 5 & cDE/rand/1/bin & 5 & DE $/$ nrand $/ 1 /$ bin \\
\hline- & - & 6 & $\mathrm{cDE} / \mathrm{rand} / 1 / \mathrm{bin}$ \\
\hline
\end{tabular}

In this study, we have included only basic MMO search techniques in the SelfMMOGA. Nevertheless, it performs well due to the effect of collective decision making in the ensemble. 
The key feature of the approach is that it operates in an automated, self-configuring way. Thus, the SelfMMOGA can be a good alternative for complex black-box MMO problems.

\section{Conclusion}

In this study, a novel genetic algorithm (called SelfMMOGA) for multimodal optimization is proposed. It is based on self-configuring metaheuristic, which involves many different search strategies in the process of MMO problem solving and adaptively control their interactions.

The SelfMMOGA allows complex MMO problems to be dealt with, which are the black-box optimization problems (a priori information about the objective and its features are absents or cannot be introduces in the search process). The algorithm uses binary representation for solutions, thus it can be implemented for many real-world problems with variables of arbitrary (and mixed) types.

We have included 6 basic MMO techniques in the SelfMMOGA realization to demonstrate that it performs well even with simple core algorithms. We have estimated the SelfMMOGA performance with a set of binary benchmark MMO problems and continuous benchmark MMO problems from IEEE CEC 2013 Special Session and Competition on Niching Methods for Multimodal Function Optimization. The proposed approach has demonstrated a performance comparable with other well-studied techniques.

Experimental results show that the SelfMMOGA outperforms the average performance of its stand-alone algorithms. It means that it performs better on average than a randomly chosen technique. This feature is very important for complex black-box optimization, where the researcher has no possibility of defining a suitable search algorithm and of tuning its parameters. The proposed approach does not require the participation of the human-expert, because it operates in an automated, self-configuring way.

In further works, we will investigate the SelfMMOGA using more advanced component techniques.

The research was supported by President of the Russian Federation grant MK-3285.2015.9.

\section{References}

[1] S.Das, S.Maity, B.-Y.Qub, P.N.Suganthan, Real-parameter evolutionary multimodal optimization: a survey of the state-of-the art, Swarm and Evolutionary Computation, 1(2011), $71-88$.

[2] M.Preuss, Tutorial on Multimodal Optimization, In the 13th International Conference on Parallel Problem Solving from Nature, PPSN 2014, Ljubljana, Slovenia, 2014.

[3] X.Li, A.Engelbrecht, M.G.Epitropakis, Benchmark functions for CEC'2013 special session and competition on niching methods for multimodal function optimization, Evol. Comput. Mach. Learn. Group, RMIT University, Melbourne, VIC, Australia, Tech. Rep., 2013.

[4] Y.Liu, X.Ling, Zh.Shi, M.Lv, J.Fang., L.Zhang, A Survey on Particle Swarm Optimization Algorithms for Multimodal Function Optimization, Journal of Software, 6(2011), no.12, $2449-2455$. 
[5] K.Deb, A.Saha, Finding Multiple Solutions for Multimodal Optimization Problems Using a Multi-Objective Evolutionary Approach, Proceedings of the 12th Annual Conference on Genetic and Evolutionary Computation, GECCO 2010, ACM, New York, 2010, 447-454.

[6] M.Bessaou, A.Petrowski, P.Siarry, Island Model Cooperating with Speciation for Multimodal Optimization, Parallel Problem Solving from Nature PPSN VI, Lecture Notes in Computer Science, 1917(2000), 437-446.

[7] E.L.Yu, P.N.Suganthan, Ensemble o niching algorithms, Information Sciences, 180(2010), no. $15,2815-2833$.

[8] B.Qu, J.Liang, P.N.Suganthan, T.Chen, Ensemble of Clearing Differential Evolution for Multi-modal Optimization, Advances in Swarm Intelligence, Lecture Notes in Computer Science, 7331(2012), 350-357.

[9] E.Sopov, A Self-configuring Metaheuristic for Control of Multi-Strategy Evolutionary Search, ICSI-CCI 2015, Part III, Lecture Notes in Computer Science, 9142(2015), 29-37.

[10] G.Singh, K.Deb, Comparison of multi-modal optimization algorithms based on evolutionary algorithms, In Proceedings of the Genetic and Evolutionary Computation Conference, Seattle, 2006, 1305-1312.

[11] M.Preuss, S.Wessing, Measuring multimodal optimization solution sets with a view to multiobjective techniques, EVOLVE - A Bridge between Probability, Set Oriented Numerics, and Evolutionary Computation IV. AISC, Springer, Heidelberg, 227(2013), 123-137.

[12] M.Preuss, C.Stoean, R.Stoean, Niching foundations: basin identification on fixed-property generated landscapes, Proceedings of the 13th Annual Conference on Genetic and Evolutionary Computation, GECCO 2011, 2011, 837-844.

[13] E.S.Semenkin, M.E.Semenkina, Self-configuring Genetic Algorithm with Modified Uniform Crossover Operator, Advances in Swarm Intelligence, Lecture Notes in Computer Science, 7331(2012), 414-421.

[14] X.Li, A.Engelbrecht, M.Epitropakis, Results of the 2013 IEEE CEC Competition on Niching Methods for Multimodal Optimization, Report presented at 2013 IEEE Congress on Evolutionary Computation Competition on: Niching Methods for Multimodal Optimization, 2013.

[15] D.Molina, A.Puris, R.Bello, F.Herrera, Variable mesh optimization for the 2013 CEC special session niching methods for multimodal optimization, Proc. 2013 IEEE Congress on Evolutionary Computation (CEC'13), 2013, 87-94.

[16] M.G.Epitropakis, X.Li, E.K.Burke, A dynamic archive niching differential evolution algorithm for multimodal optimization, Proc. 2013 IEEE Congress on Evolutionary Computation (CEC'13), 2013, 79-86.

[17] S.Bandaru, K.Deb, A parameterless-niching-assisted bi-objective approach to multimodal optimization, Proc. 2013 IEEE Congress on Evolutionary Computation (CEC'13), 2013, $95-102$. 


\title{
Идентификация множества экстремумов с помощью гене- тического алгоритма на основе многих стратегий мульти- модального поиска
}

Евгений А. Сопов

Институт информатики и телекоммуникаций Сибирский государственный аэрокосмический университет Красноярский рабочий, 31, Красноярск, 660037

Россия

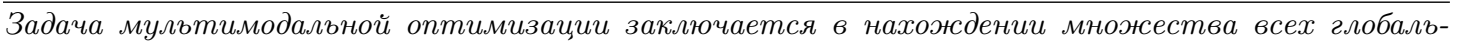
ных и локальных оптимумов или аппроксимации этого множества. В данной работе предложен новый подход, основанный на метаэвристике для построения генетического алгоритма, включающего многие стратегии поиска. Предложенный подход позволяет управлять взаимодействием нескольких поисковых алгоритмов (разных генетических алгоритмов для мультимодальной оптимизации) и обеспечивает самоконфигурируемое решение задачи с априори неизвестной структурой. В статъе представлены результаты численных экспериментов и сравнение с другими известными подходами на множестве традиционных тестовых задач мультимодальной оптимизации и задачах, предложенных на конкурсе по мультимодальной оптимизачии в рамках конференции IEEE CEC. Предложенный подход демонстрирует лучшую эфбективность, чем стандартные подходы, основанные на идее ниш, и сравнимую с современными усовершенствованными алгоритмами. Особенностъю и преимуществом предложенного подхода является то, что он не требует привлечения дополнительных экспертных знаний, так как работает в автоматизированном, самоконфигурируемом режиме.
\end{abstract}

Ключевые слова: мультимодальная оптимизачия, самоконфигурирование, генетический алгоритм, метаэвристика, метод ниш. 